Archives de sciences sociales des religions

115 | juillet-septembre 2001

Islam et politique dans le monde (ex-)communiste

\title{
Islam et politique en Asie centrale
}

\section{Olivier Roy}

\section{OpenEdition}

Journals

Édition électronique

URL : http://journals.openedition.org/assr/18303

DOI : $10.4000 /$ assr. 18303

ISSN : $1777-5825$

Éditeur

Éditions de l'EHESS

Édition imprimée

Date de publication : 1 octobre 2001

Pagination : 49-61

ISBN : 2-222-96707-4

ISSN : 0335-5985

Référence électronique

Olivier Roy, «Islam et politique en Asie centrale », Archives de sciences sociales des religions [En ligne], 115 | juillet-septembre 2001, mis en ligne le 19 août 2009, consulté le 01 mai 2019. URL : http:// journals.openedition.org/assr/18303 ; DOI : 10.4000/assr.18303

Ce document a été généré automatiquement le 1 mai 2019.

(C) Archives de sciences sociales des religions 


\title{
Islam et politique en Asie centrale
}

\author{
Olivier Roy
}

1 L'isolement de l'Asie centrale durant les quelque sept décennies de soviétisme a produit une configuration très particulière des relations entre islam, politique et vie sociale, du fait que la religion musulmane, persécutée, se soit maintenue, comme les autres religions, dans la clandestinité ou le cadre d'un clergé-croupion officiel. Mais la décennie qui suivit les indépendances de 1991 a, d'une certaine manière, réintégré l'Asie centrale dans les grands courants du monde musulman contemporain : une réislamisation conservatrice de la société, un islam officiel bien contrôlé par les gouvernements et un islam radical qui, soit rentre dans le jeu politique (Tadjikistan), soit devient l'expression d'une contestation sociale face à un régime autoritaire (Ouzbékistan), tandis que le soufisme tend à être apolitique.

\section{I - L'héritage soviétique}

2 Il convient donc de rappeler d'abord les grands traits de l'islam soviétique au moment de l'indépendance ${ }^{1}$. Se réclamant du sunnisme hanafite, fortement marqué par un soufisme très orthodoxe, coupé du Moyen-Orient après le passage de l'Iran au chiisme durant le $\mathrm{XVI}^{\mathrm{e}}$ siècle, l'islam d'Asie centrale s'est trouvé, au XIX ${ }^{\mathrm{e}}$ siècle, partagé entre une influence réformiste surtout venue des Tatars de l'empire russe et un fondamentalisme plus strict développé dans les écoles du sous-continent indien (Déoband). Paradoxalement la révolution bolchevique a porté un coup plus rude au réformisme moderniste (le jadidisme ), véhiculé par des intellectuels urbains, parfois compagnons de route du communisme et éliminés dans les purges des années trente, qu'au traditionalisme qui a trouvé un refuge chez les mollahs ruraux moins soumis à la répression. Après l'écrasement de la révolte des Basmatchis et la grande attaque frontale (hojjum) contre l'islam et la société traditionnelle en 1927, les manifestations visibles de l'islam ont connu une longue éclipse. Cependant, l'islam s'est maintenu, sous des formes évidemment différentes mais plus complémentaires qu'antagonistes, sur quatre registres, correspondant à des catégories d'acteurs différents. 


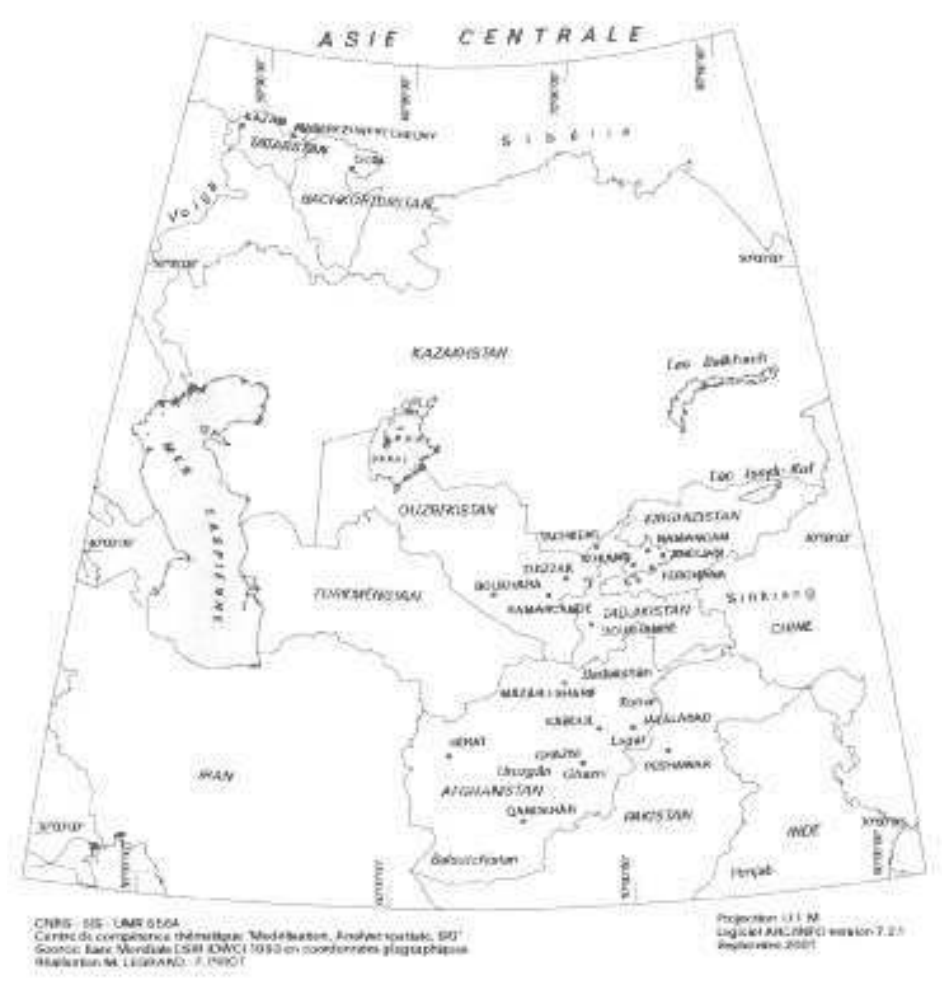

CNRS - SIS - UMR 8564

Centre de compétence thématique « Modélisation, Analyse spatiale, SIG » Source : Base Mondiale ESRI (DWC) 1993 en coordonnées géographiques Projection UTM

Logiciel ARC/INFO version 7.2.1

Septembre 2001

Réalisation : M. Legrand, F. Pirot

$31^{\circ} \mathrm{Un}$ islam parallèle, conservateur, lié à la société rurale traditionnelle telle qu'elle s'est articulée sur le système soviétique des kolkhozes, avec des poches régionales de radicalisme. Les mosquées sont fermées, mais des mollahs parallèles, dont la fonction se transmet le plus souvent de père en fils, maintiennent la tradition, tout en n'étant officiellement que de simples kolkhoziens. Agissant dans le cadre des groupes de solidarité traditionnels, ils bénéficient du silence voire de la complicité des apparatchiks locaux. Cet islam est d'ailleurs considéré par la population comme partie intégrante d'un héritage et d'une identité qui ont survécu au système soviétique (maintien des grands rites de passage, comme la circoncision, usage de noms propres musulmans etc.). Le corpus est celui en usage dans les mak-tab et madrasa d'avant la révolution et fait souvent aussi l'objet d'une transmission familiale (grand-parents apprenant à leurs enfants les rudiments de l'islam) ${ }^{2}$.

$4 \quad 2^{\circ}$ Un islam officiel, mis en place en 1941 mais rajeuni dans les années quatre-vingt: à cette époque de jeunes mollahs furent envoyés dans les pays arabes afin d'y acquérir une maîtrise de la langue et du corpus islamique pour rendre crédible l'islam soviétique. Cet islam était organisé dans le cadre d'une Direction spirituelle, basée à Tachkent, dirigée par un mufti ouzbek dont l'autorité s'étendait aux quatre pays d'Asie centrale proprement dite et au Kazakhstan. La Direction gérait les quelques mosquées restant officiellement ouvertes. À la fin des années quatre-vingt, un personnel peu nombreux 
mais jeune et compétent (Mohammad Youssouf, nommé en 1989 mufti à Tachkent, ou Akbar Touradjanzade, Qazi à Doushanbé), avait évincé la dynastie des Babakhanov dont trois générations successives ont tenu le muftiyyat de Tachkent. Lors de leurs études (respectivement en Libye et en Jordanie), ces clercs ont rencontré l'influence des Frères musulmans.

$5 \quad 3^{\circ}$ L'émergence à la fin de la période soviétique de jeunes intellectuels islamiques ayant une formation à la fois moderne et traditionnelle et qui se sont manifestés au moment de la guerre d'Afghanistan (Sayyid Abdoullah Nourov, dit Mollah Nouri, arrêté au Tadjikistan pour son soutien aux Moudjahidin afghans en décembre 1986, est ingénieur en géodésie et a suivi les cours clandestins d'un certain Mollah Hindoustani, lui-même formé en Inde dans les années vingt et rescapé des camps de Sibérie).

$4^{\circ}$ Enfin, il ne faut pas négliger le rôle des orientalistes issus de l'Asie centrale. Supposés être de bons apparatchiks athées, de jeunes Ouzbeks, Tadjiks, Kazakhs etc., ont été formés à l'arabe et au persan, dans le cadre des instituts d'études orientales, afin de servir de relais au système soviétique dans le monde musulman (comme diplomates, traducteurs, experts) dans les années 1970 et 1980 (en Afghanistan en particulier). En général loyaux au système soviétique, on les retrouvera souvent après l'indépendance comme cadres d'un nouvel islam officiel.

7 Aucun de ces acteurs ne s'est particulièrement intéressé aux questions du réformisme doctrinal (sinon un petit groupe de "wahhabi " autour de Rahmatoullah Allama, un disciple de Qazi Hindoustani). Ils sont soit très traditionalistes, soit préoccupés avant tout d'action politique. L'héritage jadid ne s'est pas transmis. C'est donc surtout à l'étranger que les acteurs du revivalisme islamique postindépendance iront chercher leurs modèles.

\section{II - Les indépendances et la ré-islamisation}

8 Ces quatre catégories d'acteurs se sont donc soudainement trouvé un nouvel espace d'action après l'indépendance, d'autant que les contacts avec le monde musulman se sont, d'un seul coup, multipliés, sous la forme de venues de prédicateurs, d'octroi de bourses ou d'envoi de littérature religieuse. Ce sont surtout les pays du Golfe et le Pakistan qui ont joué un rôle. L'Iran en effet a manqué d'une part d'un relais idéologique (absence de chi'ites en Asie centrale) et s'est efforcé de conserver de bonnes relations avec Moscou afin de contrer l'influence américaine, turque et... saoudienne. L'Iran n'a joué de rôle qu'au Tadjikistan, du fait de la proximité linguistique, en soutenant l'opposition islamiste, avant de la pousser à trouver un accord avec Moscou, ce qui fut fait en juin 1997. Enfin les développements des guerres civiles d'Afghanistan et du Tadjikistan ont brouillé les frontières et mis en contact direct les radicaux afghans et ceux d'Asie centrale. Les gouvernements des nouvelles républiques, après un certain libéralisme en 1991 et 1992, se sont lancés dans une double politique de répression de l'islam radical et de mise en place d'un islam officiel.

9 La réislamisation des sociétés après l'indépendance est un fait, même si souvent, il s'agit simplement de l'apparition au grand jour de pratiques religieuses vivantes mais demeurées discrètes. La construction des mosquées a sans doute été le phénomène le plus spectaculaire, même s'il faut faire la distinction entre les grandes mosquées " cathédrales » (jomac), par exemple dans le Ferghana, propres à offrir un cadre à une mobilisation politique plus large, les mosquées « historiques » qui permettent aux États 
de renouer avec l'histoire (le tombeau de Bahauddin Naqshband à Boukhara) et enfin des mosquées de quartier (mahalla), expression de la sociabilité locale. Il est d'ailleurs parfois difficile de distinguer ce qui relève de l'islamisation (port du voile) et d'une retraditionalisation encouragée par l'État, se traduisant par un plus grand conservatisme des mœurs et un déclin du statut de la femme. C'est ainsi qu'en Ouzbékistan, par son insistance sur la tradition, le gouvernement du Président Karimov a, de fait, encouragé une ré-islamisation qui pourrait se retourner contre lui : par exemple la loi de 1993 sur les mahalla (quartiers) en institutionnalisant le pouvoir des aqsaqal (les « anciens ») a souvent donné un poids nouveau à des mollahs parallèles. Enfin, dans leur quête de reconnaissance internationale, les nouveaux États ont été amenés à laisser le champ libre à certains pays musulmans pro-occidentaux (comme l'Arabie Saoudite) tout en s'opposant à l'Iran, ce qui a contribué à façonner le type de prédication islamique dans le sens du radicalisme sunnite.

La question de l'islam est donc un enjeu pour l'ensemble des acteurs du champ politique.

\section{$1^{\circ}$ La mise au pas et le développement du clergé officiel}

Tous les gouvernements ont repris, nationalisé et développé les structures soviétiques du clergé officiel. Malgré les velléités du chef du muftiyyat pour l'Asie centrale, Mohammed Youssouf, de maintenir une instance transnationale après 1991, chaque État s'est empressé de promouvoir le Qazi de la république (subordonné au Mufti de Tachkent à l'époque soviétique) au poste, créé à cette occasion, de Mufti de la république, autonome par rapport à celui de Tachkent. Cette rupture s'est faite chaque fois avec l'approbation du Qazi concerné : Sadikian Kamalov au Kirghyzstan (où le muftiyyat n'a été proclamé qu'en 1993), Ratbeg Nissan Bay (appelé aussi Nissanbaev, ou Nissan-bay oglu) au Kazakhstan, Nasroullah Ibadoulayev au Turkménistan, Mohammed Youssouf (Mamayoussoupof) à Tachkent et enfin Akbar Touradjanzade au Tadjikistan, même si ce dernier a voulu maintenir des liens avec son homologue de Tachkent.

Les deux derniers ont tenté de rendre le clergé officiel autonome par rapport au nouvel État, mais ont échoué. S'appuyant sur un islam sinon radical, du moins très «salafiste " (prônant un retour aux sources au-delà des traditions), ils se sont efforcés de contrôler le clergé parallèle, de nommer leurs propres imams dans les nouvelles mosquées, et de négocier avec l'État une sorte de concordat, qui passerait par une islamisation des lois et de la culture. Ils ont aussi essayé de s'assurer le monopole de l'aide étrangère, en particulier saoudienne. Mais, ce faisant, ils se sont heurtés aussi bien à l'État qu'aux mollahs ex-parallèles, peu soucieux de renoncer à leur nouvelle indépendance (et aussi désireux d'accéder directement à l'aide étrangère). En fait, la plupart des mollahs parallèles se sont bien gardés de constituer ou de rejoindre un mouvement qui se définirait essentiellement par l'islam : partout ils se sont au contraire alignés sur les choix politiques de leur clan et se sont montrés plus soucieux de consolider leur position de notables locaux. Même les quelques mollahs parallèles politisés ont montré de la méfiance envers la volonté des deux muftis d'affirmer leur leadership sur l'ensemble du clergé. Mohammed Youssouf a été violemment pris à partie, en 1991, par les "wahhabis » du Ferghana, qui l'accusaient de commercialiser les Corans offerts par l'Arabie Saoudite. $\mathrm{Au}$ Tadjikistan, Touradjanzade s'est allié aux islamistes du Parti de la Renaissance Islamique (PRI), mais ceux-ci n'ont jamais vraiment reconnu sa prééminence en termes religieux ${ }^{3}$. Bref les muftis se sont trouvés en porte-à-faux entre les États et l'islam radical. 
13 Après avoir rallié l'opposition, Touradjanzade a dû fuir en Afghanistan à la suite de la défaite du camp "islamo-démocrate " en décembre 1992; Mohammed Youssouf a été contraint à l'exil en avril 1993. Tous deux ont été remplacés par des muftis apolitiques (respectivement Fathoullah Khan Sharifzade et Hajji Moukhtar Abdoullah). Les États ont repris l'institution $d u$ "comité des affaires religieuses», instance bureaucratique dépendant directement $d u$ gouvernement et chargée de contrôler le clergé; les « orientalistes » laïques en ont souvent fourni le personnel (comme l'historien Shoazim Mounavvarof en Ouzbékistan). Enfin, partout une loi a organisé le champ religieux en étendant le contrôle du clergé officiel, une fois "normalisé", sur toutes les grandes mosquées (les mosquées "jomac» où est prononcé le prêche); pour les petites mosquées de quartiers (appelées panjwaghti ou beshwaghti, c'est-à-dire destinées uniquement aux cinq prières), le contrôle est plus souple, mais le prêche y est souvent interdit (Ouzbékistan, Tadjikistan, Turkménistan). Le hajj et les grandes institutions d'enseignement religieux sont contrôlés par l'État. En avril 1999, l'ouverture d'une université islamique d'État est annoncée à Tachkent. De même après 1993, un contrôle strict des visas a limité l'entrée en Ouzbékistan des prédicateurs étrangers.

C'est en Ouzbékistan que le contrôle de l'État est le plus développé. Les madrasa n'ont le droit de recruter qu'après l'école moyenne. Il y a 18 madrasa provinciales (une par province ou velayat, plus trois à Tachkent), prolongées par une école supérieure (Imam Boukhari) où le cursus dure quatre ans. Une commission (hayat) des oulémas présidée par le mufti est l'instance supérieure du muf-tiyyat (avec présidium de 12 à 13 membres); son interlocuteur est le comité des affaires religieuses auprès du conseil des ministres. Dans chaque velayat il y a un mufti. Tous les imam des mosquées jomac sont nommés par le muftiyyat (ou confirmés par lui pour ceux déjà en place avant la loi). Une commission de contrôle instituée en 1997 a entrepris l'enregistrement ou l'invalidation de tous les imams déjà en place, en commençant par les territoires sensibles: Tachkent, Namangan, Ferghana, Samarkand. Par contre l'État peut se montrer généreux envers le clergé ainsi domestiqué : il a donné 40 hectares de terres en « waqf » à Djizzak, le fief du président. Le $1^{\text {er }}$ mai 1998 est promulguée la loi sur la «liberté de conscience " exigeant l'enregistrement préalable de tout groupe religieux de plus de 100 personnes et de toutes les mosquées. Chaque religion doit être représentée par une seule institution pour tout le pays. L'État a ainsi obtenu le soutien complet de la hiérarchie religieuse sur le plan politique: Abdourrashid Bahrom, le dernier mufti en date, promulgue ainsi une fatwa contre le Hizb-ul-Tahrir en juin 1999.

15 Au Tadjikistan, la loi du 23 mai 1998 interdit les partis religieux (y compris le Parti de la Renaissance Islamique qui fait partie de la coalition), même si, en août 1999, la cour suprême lève l'interdiction des partis d'opposition.

Pourtant les nouveaux régimes se sont bien gardés de retomber dans un antiislamisme militant. Seuls le Kazakhstan et le Turkménistan se proclament officiellement laïques ( diinyavi) dans leur constitution. Ouzbékistan et Tadjikistan, où l'influence de l'islam est historiquement forte, reprennent volontiers à leur compte l'héritage musulman, en rendant officiellement chômées les grandes fêtes religieuses (fitr et bayram). L'Ouzbékistan a réhabilité en 1993 Bahaouddin Naqhshband, le fondateur de la naqhshbandiyya. Au Tadjikistan, le clan koulabi, après sa victoire contre les islamistes, restaure en grande pompe le mausolée de Mir Saïd Ali Hamadani, pour son $480^{\mathrm{e}}$ anniversaire, tandis que l'Académie des Sciences publie en 1994 « Ta'alimat-i sufizm dar ijadiat-i Ali-ye Hamadani» («L'enseignement du soufisme dans les œuvres d'Ali 
Hamadani »). Les présidents n'hésitent pas à aller faire au moins le petit pèlerinage à la Mecque, et tous les pays d'Asie centrale ont adhéré à l'Organisation de la Conférence islamique.

17 Les États ont donc gagné le contrôle de l'islam officiel, selon une politique qui rappelle celle du Maroc, de l'Égypte et de la Turquie (le modèle du Diyanet a d'ailleurs été systématiquement proposé par la Turquie aux nouveaux États).

\section{$2^{\circ}$ Le clergé parallèle}

18 Le clergé parallèle s'est "professionnalisé » dès l'indépendance: on abandonne son métier officiel pour tenter de vivre en tant que mollah, soit de dons, soit d'un petit salaire officiel en entrant dans le clergé officiel. Souvent le mollah s'est transformé en entrepreneur pour gérer la mosquée (re)construite et l'éventuelle activité économique y afférant (offrandes, prestations pour les rites de passage). Les prédicateurs ou bienfaiteurs étrangers ont joué un rôle, car, en 1991, quelques centaines de dollars pouvaient faire beaucoup. Les mollahs parallèles, lorsqu'ils ont été contraints de faire des choix politiques, ont presque tous suivi leur clan ou leur groupe de solidarité, indépendamment de leurs convictions religieuses. L'exemple type est celui des Gharmis et des Koulabis au Tadjikistan, termes désignant deux factions localistes. Alors que la région de Koulab était célèbre sous l'époque soviétique pour le conservatisme religieux de ses habitants, presque tous ses mollahs ont rejoint en 1992 le « Front populaire » expression des apparatchiks ex-communistes contre le Parti de la Renaissance Islamique. Inversement les apparatchiks communistes de Gharm ont presque tous rejoint l'opposition islamique, au point que, dès les manifestations d'avril 1992, le terme de Koulabi était synonyme de « néocommunistes » et celui de "Gharmis » d'« islamistes ».

19 Ailleurs, les mollahs parallèles, même fondamentalistes, ont accepté un compromis avec les nouvelles autorités religieuses. Dans la ville de Kasansoy (peuplée de Tadjiks mais située dans le Ferghana ouzbek), un noyau très actif de mollahs parallèles s'est développé sous le régime soviétique, autour d'un médecin, invalide de guerre, Makhtoum, et de Jora Akhound, mort en 1988, naqshbandi'. Dès l'indépendance, l'un d'entre eux, Azzam Khan Khwadja, a entrepris de construire ce qui est sans doute la plus grande mosquée contemporaine d'Asie centrale. Le gouvernement, méfiant, a refusé de l'enregistrer comme mollah officiel, mais a accepté la candidature du numéro deux de la mosquée, Sharif Djan, né en $1954^{5}$.

20 Par contre, quelques mollahs parallèles, en général jeunes, et plus proches du modèle du militant islamiste dont nous parlerons ensuite, ont ouvert des mosquées « idéologiques » aux alentours des grandes villes et attirent des fidèles non pas parce qu'ils viennent du mahalla (le quartier) mais par attrait pour des prêches beaucoup plus incisifs et vigoureux. C'est le cas de la mosquée Djami d'Andijan. Ces mosquées, particulièrement actives dans le Ferghana, ont toutes été fermées à partir de 1995. De même, des réseaux de mollahs parallèles ont développé des activités de prêches itinérants, souvent accompagnées par des femmes prédicatrices, les otin ${ }^{6}$. Ils interviennent par exemple dans les fêtes de mariages ou de circoncisions (les tuy), insistant pour qu'alcool, musique et mixité en soient bannis. Des organisations internationales islamiques (Rabita, Tabligh) proposent bourses et documentation : des jeunes, revenus à l'islam, peuvent ainsi partir à l'étranger et, à leur retour, se posent à leur tour en mollahs, de manière tout à fait ouverte. 
21 On trouve aussi des synthèses entre mollahs parallèles et acteurs modernes, comme l'association entre un club de sport de combat et une mosquée, qui semble avoir existé à Namangan dans le Ferghana au moment des soulèvements islamiques de 1992. En mai 1997, on annonce la fermeture à Tachkent d'un club de kick-boxing (dont l'entraîneur est un certain Fathoullaïev) du fait de l'activisme religieux de ses membres ${ }^{7}$.

22 Tous ces indices montrent le développement récent d'un nouveau clergé parallèle, plus jeune, plus urbain, plus éduqué, où se rejoignent la tradition du clergé parallèle et la mouvance islamiste récente. Ce sont eux que l'on appelle les «wah-habis ». C'est dans cette mouvance que se développe depuis 1996 un parti politiquement difficile à classer : le Hizb-ul-Tahrir, ou "parti de la libération », fondé par Taqieddin Nabhani en 1953 en Jordanie, aujourd'hui dirigé depuis Beyrouth par Abdel Qadim Zaloum, depuis la mort de Nabhani en 1977. Un autre centre se trouve à Londres, dirigé par Omar Bakri Mohammed, syrien, émigré dans les années quatre-vingt, qui a participé au Congrès du califat à Londres le 7 août 1994. Créé comme parti palestinien islamo-nationaliste, le Hizb-ulTahrir a évolué peu à peu en un mouvement supra-national, travaillant pour le rétablissement du Califat. Il recrute surtout parmi la seconde génération issue de milieux immigrés en Europe. Verbalement très agressif, refusant tout compromis non seulement avec les pouvoirs en place mais aussi avec les musulmans qui ne reconnaissent pas son leadership, il n'a cependant pas recours à la violence armée ou au terrorisme, mais prône la « reconversion » des musulmans « égarés » ou assoupis, grâce à la prédication. Le Hizbul-Tahrir a fait une percée inattendue parmi la jeunesse ouzbèke, y compris parmi les Ouzbeks du sud-Kirghyzstan. Les rares documents émanant de la branche ouzbèke utilisent la même terminologie que le mouvement basé à Londres et Beyrouth. Il fait allégeance au Cheykh Zaloum, et reprend la constitution du "Califat » telle qu'elle a été formulée en 1979 par le parti ${ }^{8}$. À l'intersection entre secte et mouvement politique, ce mouvement correspond nettement à une rupture avec les formes de religiosité traditionnelle et à une connexion sur les réseaux transnationaux.

\section{$3^{\circ}$ Le soufisme}

On sait qu'Alexandre Bennigsen voyait dans les soufis un des piliers de l'islam clandestin à l'époque soviétique 9 . On peut s'étonner de ne les voir qu'en passant dans un article consacré aux relations entre islam et politique en Asie centrale. Si le prestige et la rémanence du soufisme sont indéniables, à la fois par le poids des références soufies dans la culture musulmane d'Asie centrale et par le respect accordé aux grandes familles soufies qui ont pu survivre, l'impact politique du soufisme est faible. D'abord parce qu'il n'a pas été ou n'est plus une forme d'organisation structurée en Asie centrale. Ensuite parce que sa diffusion générale fait que tout le monde est plus ou moins lié à une grande famille soufie, ce qui enlève toute pertinence politique au fait soufi. Les liens qui unissent encore pir et mourid (très distendus par l'affaiblissement des pratiques de zikr et d'initiations sous le soviétisme) ne recoupent pas les affiliations politiques. Au Tajikistan, le Qazi Touradjanzade est le fils d'un mourid qadéri très respecté qui n'a jamais été inquiété par le régime néo-communiste après l'exil de son fils. De même le Qazi avait un grand respect pour le père (Damollah Mohammed-e Sharif Hissari) de son successeur, Fathoullah Khan Sharifzade, lequel avait été pourtant nommé par les néo-communistes. Le Cheykh Hissari avait d'ailleurs des disciples aussi bien parmi les Ouzbeks du Ferghana que parmi les Tadjiks du sud du Tadjikistan. Lorsque le Pir Ishan Abdourrahman Djan de 
Ab-i Garni, un Naqshbandi, est enterré le 12 juillet 1991, il est loué aussi bien par le PRI que par Kenjaiev qui sera l'adversaire déterminé du parti islamiste lors de la guerre civile ; le fils du pir, Hajji Abdoulqouddous, soutient Touradjanzade, alors que son neveu, Ne'matzadeh, serait devenu le troisième mufti " néo-communiste ", après l'assassinat de Sharifzade. Bref, les grandes familles religieuses, ou plutôt ce qu'il en reste après le soviétisme, ont un rayonnement qui coupe à travers les allégeances politiques (et aussi, ce qui va de pair, à travers les allégeances ethniques et régionalistes). Elles n'ont pas en soi de rôle politique et ne correspondent pas à des réseaux structurés.

D'autre part, selon un phénomène courant aussi en Afghanistan, les descendants de pir prestigieux ne sont pas nécessairement eux-mêmes de grands religieux, ce qui affaiblit la transmission, ou plutôt fait du respect qu'on porte à ces descendants une sorte de convenance sociale et non un lien de maitre à disciple. Le retour au soufisme que l'on observe aujourd'hui relève plus d'un phénomène de néo-confrérisme, où on réinvente de manière autodidacte des pratiques de soufisme, tout en ayant perdu la véritable transmission. Enfin, il est de bon ton aujourd'hui, dans les milieux gouvernementaux, surtout ouzbeks, de mettre le soufisme en avant, parce qu'il incarnerait un islam «national » et anti-wahhabi ${ }^{10}$.

Certains « wahhabis » commencent à critiquer ouvertement les pirs soufis. B. Babadjanov cite ainsi une polémique à Andijan où un des rares authentiques pir naqshbandis, Hazrat Ishan Ibrahim, de Kokand, s'est fait vertement interpeller par de jeunes mollahs formés en Arabie Saoudite ${ }^{11}$. Les tentatives de relancer le soufisme, souvent faites par des néophytes, vont à l'encontre de la prédication «wahhabie» et tendent donc à être apolitiques.

\section{$4^{\circ}$ Les mouvements islamistes et les « wahhabis »}

La radicalisation politique de jeunes intellectuels a commencé bien avant la dissolution du régime soviétique. Un personnage clé $^{12}$ a joué un grand rôle : Mawlawi Qari Hindoustani dit aussi Hajji Mohammad Roustamov ou Qari Mohammed Jan Damolla (né en 1892 à Kokand, mort en 1989 à Douchanbé). Ouzbek du Ferghana, formé en Afghanistan et au Cachemire, écrivant en persan, ayant fait 15 ans de prison en URSS, il établit dans les années soixante-dix une madrasa clandestine à Douchanbé, avant d'acquérir un statut semi-officiel dans les années 1980. Son influence va de l'Ouzbékistan au Tadjikistan et ses élèves sont indifféremment Ouzbeks et Tadjiks. Il a formé la plupart des acteurs islamistes des années 1990 (Allama Rahmatoullah, Mollah Nouri, Himmatzadeh, Damollah Hikmatoullah), qui l'ont quitté à la fin des années 1970, lui reprochant son refus de soutenir les Moudjahidines afghans. À l'inverse, il les accusa alors de wahhabisme, c'est-à-dire de déviation par rapport au sunnisme hanafite ${ }^{13}$. On les retrouvera également au parti de la Renaissance islamique.

L'islamisme, au sens où on l'entend usuellement (mouvement mené par des intellectuels de formation moderne et voyant dans l'islam une idéologie politique à même de permettre l'établissement d'un «État islamique »), apparaît en URSS avec le « Parti de la renaissance islamique $»^{14}$. Fondé à Astrakhan en juin 1990, sous la direction de Ahmedzay Akhtaev (Avar du Daghestan) et Valiahmad Sadour (Tatar de Moscou, spécialiste d'indonésien), il se présente comme un mouvement supranational, apte à fédérer les musulmans sur l'ensemble du territoire soviétique. La direction de Moscou s'oppose d'abord aux indépendances, ce qui entraîne rapidement la scission des sections tadjike 
(novembre 1991) et ouzbèke. C'est au Tadjikistan que le parti connaît son développement le plus spectaculaire, puisqu'il constitue la colonne vertébrale de l'opposition qui prend le pouvoir en mai 1992. Le parti y est dirigé par Mohammadsharif Himmatzadeh et Dawlat Osman, mais Mollah Nouri joue un rôle important.

Nous ne reviendrons pas ici sur la guerre civile au Tadjikistan qui a fait l'objet d'une littérature importante, mais sur la période qui a suivi. L'évolution du PRI tadjik entre son exil en Afghanistan (décembre 1992) et son retour à Douchanbé dans le cadre d'un accord avec le régime "néo-communiste " pour un gouvernement de coalition (juin 1997) est tout à fait conforme à l'évolution «islamo-nationale» de la plupart des grands mouvements islamistes du Moyen-Orient (Hamas, Hizbullah, FIS, Frères musulmans, Refah/Fazilet etc. ${ }^{15}$. Le mouvement inscrit son action dans un cadre avant tout national et met une sourdine à la revendication d'un État islamique. Ses alliances ont à voir avec l'intérêt national tel qu'il le conçoit et pas avec les affinités idéologiques. Tout d'abord le PRI a toujours agi dans le cadre d'une alliance avec les démocrates du mouvement « Rastakhiz» et la minorité ismaélienne du Pamir, tous deux très laïques. L'Opposition Tadjike Unifiée (UTO) a toujours présenté un front commun dans les négociations. Lors de son exil en Afghanistan, le PRI (qui s'est alors appelé "Mouvement islamique») s'est trouvé dans des zones tenues soit par Massoud (Taloqan), soit par des fondamentalistes proches des milieux radicaux arabes et pakistanais (Kunduz) ou bien chez le Général Doustom (Mazar-i Sharif). À Kunduz, une organisation de solidarité islamique, l'IIRO, à fonds saoudiens, joua un grand rôle en mettant en contact les islamistes d'Asie centrale avec les réseaux internationaux de formation et de soutien. Le PRI a été alors vivement soutenu par les réseaux internationalistes arabes et pakistanais installés dans la région de Lahore $^{16}$. Les 8 et 10 novembre 1995, le Jamacat-i Islami pakistanais organise un grand rassemblement islamiste international à Lahore : le PRI est représenté par Himmatzadeh.

Lors de l'offensive des Taliban sur Kaboul (1996) et le ralliement de la poche de Kunduz aux Taliban, le PRI doit faire un choix : soit l'internationalisme militant et le ralliement aux Taliban, qui l'encouragent à la lutte armée jusqu'au bout, soit la solution nationale, autour de l'identité tadjike, à laquelle pousse le commandant Massoud, qui a besoin du soutien d'un Tadjikistan stable. Juste après la signature de l'accord de Moscou en juin 1997, qui entérine la solution nationale, sous l'égide de Moscou, Téhéran et Massoud, les Taliban interceptent l'avion qui ramène Nouri à Douchanbé depuis Téhéran (octobre). Ils tentent de le convaincre de reprendre le jihad à partir des territoires afghans qu'ils contrôlent. Nouri refuse. Les militants de l'UTO rentrent au Tadjikistan à partir du territoire afghan tenu par Massoud. Depuis, leur discours est centré sur la défense de l'identité tadjike contre la menace ouzbèke. Bien sûr l'islam n'est pas oublié, mais la revendication de l'islamisation est reformulée à partir de la défense d'une identité nationale ${ }^{17}$. Cette évolution va de pair avec celle du gouvernement de Douchanbé qui doit faire face, à partir de 1995, à une révolte dans ses rangs menée par des commandants d'ethnie ouzbèke (Mahmoud Khodaberdaïev), appuyée en sous-main par Tachkent : ici aussi les liens ethniques l'emportent sur les solidarités idéologiques. Bien sûr les choses ne se font pas simplement : certains commandants du PRI, installés dans la haute vallée de Gharm, continuent un temps la lutte armée et hébergent des militants ouzbeks. Mais la conséquence de cette évolution du PRI tadjike est la fin de la solidarité militante avec les islamistes ouzbeks, dont le destin va alors diverger. le poids de son homologue tadjik. Il a fait l'objet d'une répression précoce. Son chef, 
Abdullah Outaev, arrêté en décembre 1992, a disparu depuis, de même que Sheykh Abdoul Vali, imam de la mosquée Jami d'Andijan, et de son adjoint Ramazanbegi, en août 1995. D'autres mouvements ont aussi existé au début de l'indépendance, comme Adalat en 1992 à Namangan. Contraintes à l'exil en Afghanistan, ces différentes tendances fusionnent dans le Mouvement Islamique d'Ouzbékistan, qui se lance dans la lutte armée, sous la direction de Tahir Yoldashev et Djoma Namangani. Des troubles éclatent à Namangan en décembre 1997 (un chef de la police assassiné). Le 16 février 1998, le ministre ouzbek des affaires étrangères, A. Kamilov, dénonce le rôle joué par le Pakistan dans le soutien et l'entraînement des activistes islamiques. Un an plus tard, la crise culmine avec l'attentat contre le Président Karimov (16 février 1999) immédiatement attribué aux islamistes. En août 1999, le MOI (Mouvement Islamique d'Ouzbékistan), bien retranché en Afghanistan sous la protection des Taliban, lance des opérations armées à partir du Tadjikistan et du Kirghyzstan en visant le Ferghana. Des commandos sont même arrêtés dans les environs de Tachkent. La «menace islamiste » devient en Ouzbékistan prétexte à une répression généralisée contre toute opposition.

31 Les deux grands mouvements islamistes d'Asie centrale ont choisi des voies différentes. On retrouve, chez les islamistes, la même polarisation ethnique que chez les anciens communistes (Tadjiks contre Ouzbeks). Les Tadjiks sont dans un processus islamonationaliste, tandis que les Ouzbeks sont liés à la nébuleuse afghano-pakistanaise, soutenue par des milieux arabes ${ }^{18}$. Cette mouvance est désormais systématiquement qualifiée de «wahhabi » par les gouvernements, mais les intéressés la récusent au profit du terme « salafiste ». En fait, l'usage du mot wahhabisme reprend une tradition propre à l'époque tsariste et coloniale britannique : tout réformateur fondamentaliste était qualifié de wahhabite, surtout quand il menait campagne contre les sites soufis, ce qui est de plus en plus le cas des nouveaux militants (mais pas du PRI). Mais aujourd'hui, avec le recul de l'islamisme politique, au profit d'un néo-fondamentalisme radical qui, à l'instar des Taliban, met l'accent sur la charia et non sur la construction de l'État, il est clair que les convergences s'accentuent entre ces mouvements "néo-fondamentalistes ${ }^{19}$ et le wahhabisme saoudien, d'autant que le système des madrasa où sont formés les militants a été largement « wahhabisé » par l'argent saoudien. Ce qu'ils ajoutent au modèle saoudien, c'est l'insistance sur le djihad. Salafisme et djihadisme sont, avec l'internationalisme, les principales caractéristiques de ces réseaux, nés dans les mouvements de soutien aux Moudjahidins afghans.

32 Reste à savoir dans quelle mesure ces mouvements seront capables de sortir de leur base régionale et de mobiliser une jeunesse paupérisée et déçue par les régimes en place : le Hizb-ul-Tahrir semble mieux placé qu'eux pour cela. Mais, nous constatons que les clivages ethnico-nationaux l'emportent sur les adhésions idéologiques, y compris chez les islamistes. L'ethnicité, reconstruite par les indépendances, va sans doute rester pour longtemps la véritable clé des mouvements populaires. C'est d'autant plus vrai que les mouvements radicaux islamistes sont absents des trois autres républiques d'Asie centrale (Kazakhstan, à part un petit mouvement Alash Orda, Kirghyzstan, Turkménistan). Au Kirghyzstan, l'islamisme semble recruter dans la minorité ouzbèke d'Osh. En fait, ces mouvements « wahhabis » ne sont déstabilisateurs que dans la mesure où ils donnent une cohérence idéologique à des mobilisations ethnico-nationales et les branchent sur des réseaux internationaux susceptibles de fournir financement et soutiens. 


\section{NOTES}

1. La bibliographie est désormais abondante à ce sujet. On consultera les œuvres d'Alexandre BENNigSEN et Chantal LEMERCIER-QUelqueJAy, L'Islam en URSS, Paris, Payot, 1968 et Le Soufi et le commissaire, Paris, Le Seuil, 1986. Depuis, des travaux novateurs ont bénéficié de l'ouverture des archives soviétiques; Adeeb KHALID, The Politics of Muslim Cultural Reform, Jadidism in Central Asia, Berkeley, The University of California Press, 1998 ; Stéphane DUdoIGnon, dir., L'Islam de Russie, Paris, Maisonneuve et Larose, 1997. Pour une analyse des transformations suivant l'indépendance: Olivier RoY, La nouvelle Asie centrale ou la fabrication des nations, Paris, Le Seuil, 1997.

2. Voir KHALID, op. cit., pp. 20 sq.

3. L'éviction de Touradjanzade du PRI en octobre 1999 montre que le parti n'a en fait jamais accepté sa prétention à être le chef de l'opposition.

4. La résistance armée aux Soviétiques, menée par Nasser Khan Tora, fils de Sayyed Kamal Khwadja, a duré jusqu'au milieu des années trente.

5. De manière étonnante, l'entrée de la mosquée porte un vers qui pourrait être de Khayyam et n'a rien d'islamique: "Jihân jâm wa falak sâqi adjal $\hat{\imath}($ ?) " « le monde est une coupe et le destin l'échanson du dernier jour ».

6. Habiba FatıH, "Otines: the Unknown Women Clerics of Central Asian Islam », Central Asian Survey, vol. 16, n 1, mars 1997.

7. BBC du 6 mai 1997.

8. Pour les textes ouzbèkes, voir The Cyber Caravan, John Hopkins University, vol $1, \mathrm{n}^{\circ} 8$, mai 1999 ; pour les textes du parti, lire Suha TAJI-FARouki, A Fundamental Quest, Londres, Grey Seal, 1996, appendice.

9. Alexandre BENNIGSEN et Chantal LEMERCIER-QUELQUEJAY, Le Soufi et le commissaire, op. cit.

10. On trouvera un point sur la question dans Bakhtyar BABADJANOV «Le renouveau des communautés soufies en Ouzbékistan », in Boukhara la Noble, Cahiers d'Asie Centrale, Edisud, 1998.

11. BABADJANOV, ibid.

12. On trouvera une analyse complète et précise du personnage et de son action dans le chapitre écrit par Bakhtyar BABADJANOV «Muhammadjan Hindustani and the beginning of the "great schism » among Muslims in Uzbekistan », publié dans Islam and Politics in Russia and Central Asia ( Early I8th-Late 20th Centuries) Stéphane A. DUdoIGNON et Hisao KomATSU, dir., Londres, Kegan Paul International, 2001.

13. Si Allama Rahmatoullah (mort dans un accident de voiture en 1979) semble avoir été le plus radical dans son opposition à Hindoustani, Mollah Nouri parle avec faveur de son ancien maître et refuse l'étiquette « wahhabie » (interview personnel à Islamabad en octobre 1994).

14. Cf. Olivier RoY, op.cit.

15. Olivier RoY, Vers un islam européen (chap. 2), Paris, Esprit, 1999 (coll. « Société »).

16. On ne rentrera pas ici dans les détails de ces réseaux. Il suffit de mentionner la «jointventure " qui s'est formée au moment de la guerre d'Afghanistan entre les partis religieux pakistanais (Jama'at-i islami, Jamiat-i Ulama Islam), les services secrets pakistanais (ISI), les Saoudiens (le Prince Turki) et les Frères musulmans arabes, le tout avec la bénédiction des Américains, pour envoyer des volontaires se battre auprès des Moudjahidin afghans. Dans la décade 1990, ces réseaux se sont radicali-sés et ont entrepris de soutenir tous les Djihad, aussi 
bien contre les nouveaux régimes en Asie centrale que contre les Occidentaux. On retrouve parmi eux Oussama Bin Laden.

17. Voir l'article écrit par touRAdjanzade, « Religion: The Pillar of Society ", in Ronald SAgdeEv, Susan EISEnHower, eds., Central Asia: Conflict Resolution and Change, Washington, The Center for Post-Soviet Studies, 1995.

18. Malgré les efforts des autorités saoudiennes, désormais hostiles à cette mouvance, des transferts de fonds ont régulièrement lieu à partir de milieux d'affaires du Golfe vers Oussama Bin Laden.

19. Olivier Roy, L'Échec de l'Islam politique, Paris, Le Seuil, 1992.

\section{RÉSUMÉS}

L'Islam d'Asie centrale, durant la période soviétique, s'est structuré autour du clergé officiel et du clergé parallèle. Des mouvements plus radicaux et politisés sont apparus dès la fin des années 1970. Après les indépendances, les différents régimes ont repris et développé l'institution du clergé officiel, pour mieux contrôler la vague de réislamisation qui a submergé le Tadjikistan et l'Ouzbékistan dans les années 1990. L'islam politique s'est incarné au Tadjikistan dans le Parti de la Renaissance Islamique et en Ouzbékistan dans le Mouvement Islamique d'Ouzbékistan, engagés dans la lutte armée. Mais les deux mouvements ont divergé : les Tadjiks sont rentrés dans une logique politique et nationaliste (accord de coalition de 1997), tandis que les Ouzbeks, réfugiés auprès des Taliban en Afghanistan, mènent la guérilla contre le régime du président Karimov. Enfin le Hizb-ul-Tahrir, mouvement militant mais non-armé, basé à Londres, fait en Ouzbékistan une percée significative dans la jeunesse.

During the Soviet period, Islam in Central Asia was organized around the official and the unofficial clergy. More radical and politicized movements appeared by the end of the 1970s. After gaining their independence, the various regimes took over and developed the official clerical institution so as to control the wave of re-islamization which submerged Tadzhikistan and Uzbekistan in the 1990s. Political Islam is repre-sented by the Tadzhik Party of Islamic Renewal and the Islamic Movement of Uzbekistan, both involved in armed struggle. However, the two movements have followed separate ways. The Tadzhiks are following a political and nationalist path (coalition agreement of 1997), while the Uzbeks, having taken refuge in the region of Afghanistan controlled by the Talibans, have launched a guerilla warfare against the regime of President Karimov. Finally, the militant but non-violent movement Hizb-ul-Tahrir, based in London, is experiencing a significant breakthrough among the young generation in Uzbekistan.

El Islam de Asia central, durante el período soviético, se estructuó alrededor de un clero oficial y de un clero paralelo. Movimientos más radicales y politizados apare-cieron a partir de fines de los años '70. Luego de las independencias, los diferentes regímenes retomaron y desarrollaron la institución del clero oficial, para controlar mejor la ola de reislamización que sumergió Tadjikistan y Uzbekistán en los años '90. El Islam político se encarnó en Tadjikistan en el Partido del Renacimiento Islámico, y en Uzbekistán en el Movimiento Islámico de Uzbekistán, comprometidos en la lucha armada. Pero estos movimientos tomaron diferentes rumbos : los Tadjiks ingresaron en una lógica política y nacionalista (acuerdo de coalición de 1997), mientras que los Uzbeks, refugiados entre los Talibanes en Afganistán, conducen la guerrilla contra el 
régimen del presidente Karimov. Por último el Hizb-ul-Tahrir, movimiento militante pero no armado, con base en Londres, penetra signiflcativamente entre la juventud en Uzbekistán.

\section{AUTEUR}

\section{OLIVIER ROY}

Directeur de recherches au CNRS 